\title{
Palm print Biometrics using Feed Forward Back Propagation Neural Network
}

\author{
Rashmi Shrivas \\ School of Engineering \& IT, \\ Aarang, Raipur, India
}

\author{
Nilmani Verma \\ School of Engineering \& IT, \\ Aarang, Raipur, India
}

\author{
Vikas Singh \\ ABV- Indian Institute of \\ Information Technology and \\ Management, Gwalior, India
}

\begin{abstract}
A biometric system provides more efficient and reliable means of identity verification. By this biometric verification it can be decided whether print based identity verification system has been widely used in various applications like access control, two characteristics belongs to the same person or not. Human palm print images contain some unique objects that can easily identify people, which make it a very competitive topic in biometric research. This paper proposes a new method for human identification using palm print based biometrics. In this approach, first of all, the images of the palm are to be captured using digital camera. Then extract some special features for palm print images of human using image processing. Here special region based features are extracted that are more efficient. Dataset is to be created for using such features. Neural network is used here for the similarity matching. Back propagation algorithm is used here.
\end{abstract}

\section{KEYWORDS}

Neural network, back propagation, features extraction.

\section{Introduction and Literature Review}

Biometrics is the most secure and reliable tool for the identification and recognition of human, because it can't be stolen, but it can be borrowed. It is always associated with users. Many features of human body can be used as the purpose of biometrics based application. These may be physical like, face, finger palm or may be either behavioral like signature, speech and gait. These biometrics based identification are more secure than conventional information passing method, because there is no information to be exchanged between servers.

These are some security parameter that is associated with biometrics.

- Biometrics is based on the uniqueness of human features and we are considering similarity is null.

- Because biometrics is associated with individual so it can't be shared with other.

- Biometrics properties cannot be loss, until the serious accident.

- It can't be copied.

- So these things make biometrics more important as compare to conventional identification method.

Palm print based human identification has some advantages over other such as stable line features, rich texture features, low-resolution imaging, low-cost capturing devices, etc. [2]
[3]. So, personal identification based on palm print has become an active research topic.

There are simple principles of biometrics technology. In all biometrics based identification there are two phases, first phase will be known as enrollment phase where the individual data is to be stored in biometrics system, for this purpose scanner or camera can be used that will store biometrics features in two system, once the features are stored, the first phase is completed. Second phase is known as verification phase, where the features of the user matched with the previously stored database. If the data will match with the previously stored database, the user will be authentic or it will be identify, and will have the permission to access.

So the flow diagram for this process can be given as:-

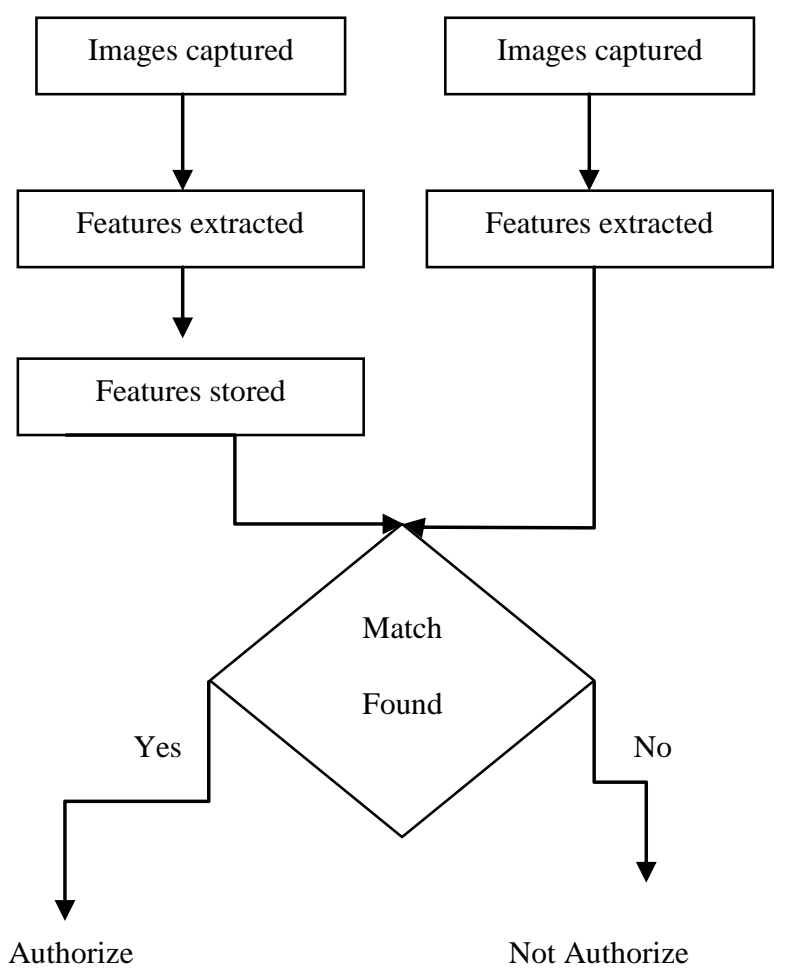

Fig. 1 Biometrics System

Many methods has been developed for biometrics based identification, these methods can be roughly classified in two categories: First one is structural based method and second one is statistical features based method. 
In structural feature based methods [4] [5] [6] structural information, such as principle lines, wrinkles, minutiae points etc., can be used as features of palm. Although line features can be detected even in the low-resolution palm print images, this kind of methods have to spend much more computation cost on matching the line segments with the templates stored in database [7]. In statistical feature based methods, palm print image is considered as a whole and palm print features are extracted by transforming the image. The extracted features are consequently used for classification. Many feature extraction methods, such as Fourier transform [8], Gabor filters [9], Eigen palm [10], fisher palm [11], independent component analysis (ICA) [4], have been explored. Except Euclidean distance [10], Hamming distance [1] etc, radial basis function neural network [12], probabilistic neural network [4] are used for feature classification.

In paper[10], robust palm print method has been describes. Here region of interest is described to find out by silent point based method. Then on region of interest the ICA method is applied to extract the features. After extracting the features the polynomial neural network is applied to classification.

In paper [11], Research of Palm print Identification Method Using Zernike Moment and Neural Network is given. In this approach given two levels of features, first one geometric feature based on distance, and second one is textures features based on Zernike Moment. Then they use the two different kind of neural and at last combined into one for effective recognition.

The rest of the paper is organized as follow. Section 2 describes the proposed system. Neural network structure for classification is described in section 3. Section 4 given the experiments and results. At last conclusion are given in section 5 .

\section{PROPOSED SYSTEM}

\subsection{Over all system approach}

Our biometrics palm print based recognition system consists of many modules. First module is image acquisition which reads image; here we are applying some preprocessing work to highlight the features of the images. Third module is feature extraction, fourth is matching, and fifth is decision.

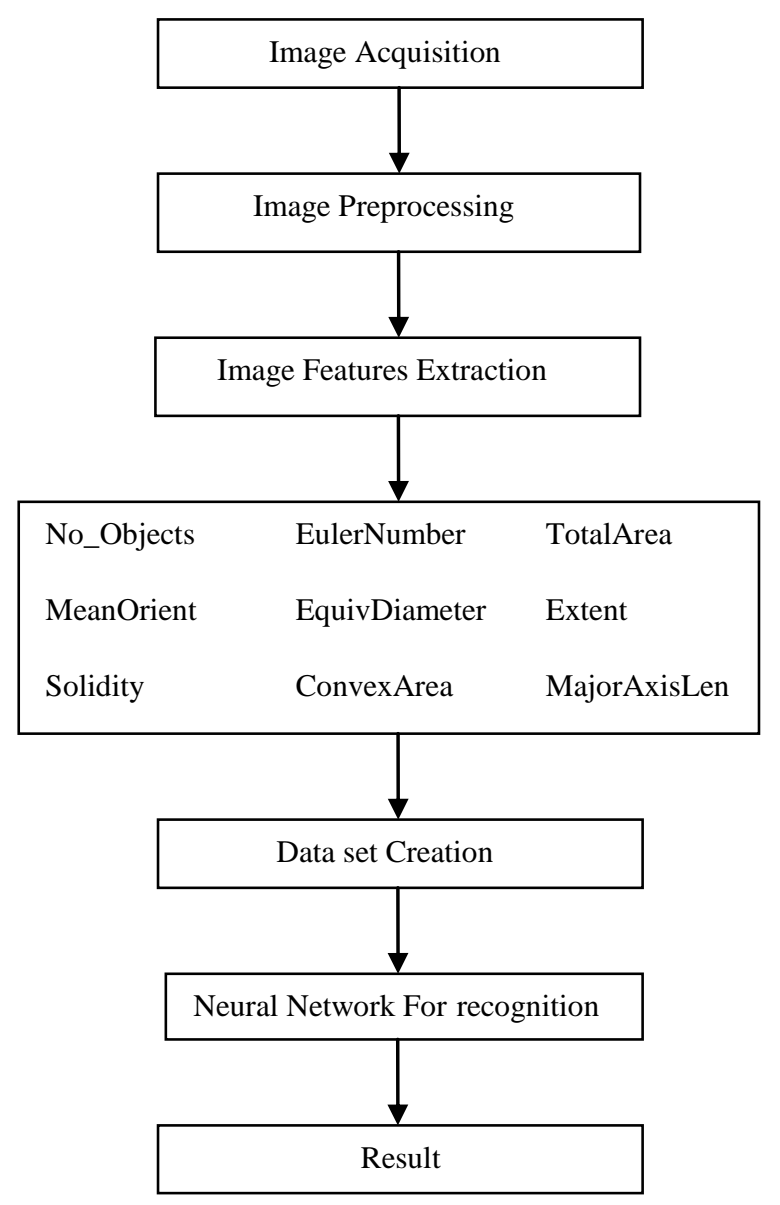

Fig. 2 Recognition system

\subsection{Image Acquisition}

This is the very important phase in any biometrics based recognition system, because the data are collected in the form of images. This phase involves the capturing and storing digital images from vision sensors. For this purpose we can use color digital cameras, monochrome and color CCD cameras, video cameras, scanners etc. The image acquisition system comprises of a light source, a digital camera/scanner. In this proposed system images are acquiesced through a digital camera. The sample database image is given below.
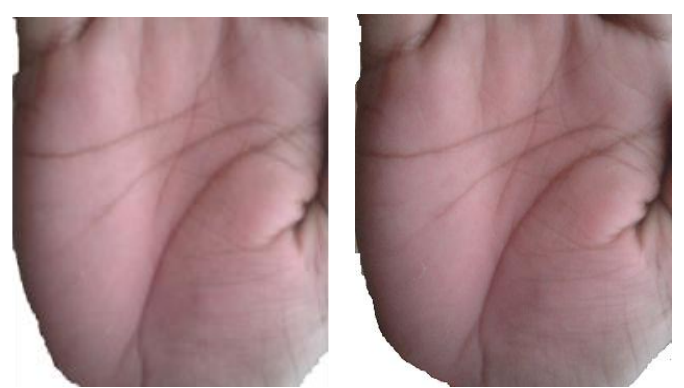

Fig.3. sample database 


\subsection{Image preprocessing}

The next phase is image preprocessing module. This phase relates to the preparation of an image which includes Conversion to Gray scale, Applying Thresholding to heightening the features. The images captured from camera do not contain clear features, so features are to be highlighted for good features extraction. After applying this image preprocessing we get the images like this:-
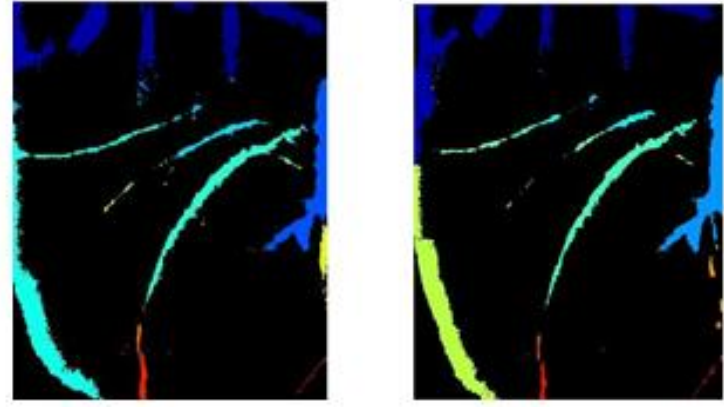

Fig. 3. Images after pre processing

\section{$2.4 \quad$ Features extraction}

The next module of biometrics palm based recognition is features extraction. Here image processing toolbox of matlab is used for this purpose. Various properties of palm print images are used as a feature here. In our work the following features are used for recognition.

- No_objects:- This function is use to calculate the different number of object present in palm region in scalar form.

- TotalArea:-This function is use to calculate the actual number of pixel in the region.

- EulerNumber:-This function specifies the number of objects in the region minus the number of holes in those objects.

- EquivDiameter:-This function specifies the diameter of a circle with the same area as the region. Computed as $\operatorname{sqrt}(4 * \mathrm{Area} / \mathrm{pi})$.

- Extent: - This function specifies the proportion of the pixels in the bounding box that also in the region. Computed as the Area divided by the area of the bounding box.

- Solidity:-This function specifying the proportion of the pixels in the convex hull that are also in the region. Computed as Area/ConvexArea.

- ConvexArea:-This function specifies the number of pixels in 'Convex Image'.

- MajorAxisLength: - This function specifying the length (in pixels) of the major axis of the ellipse that has the same normalized second central moments as the region.

- Mean orient:- This function calculate the mean of all orientation

\subsection{Data set creation}

After extracting the features datasets are created. Those are the set of numerical values for the extracted features for the different object images of the palmprint of the different person.

\section{NEURAL NETWORK STRUCTURE FOR CLASSIFICATION}

Neural network is used as classifier for the recognition purpose. Here BP neural network is used. It revises the connection power and the threshold value through the interpolation of expectation output and the actual output. It enables the error signal to achieve smallest. The prominent merit of BP network is the ability of very strong non-linear mapping and the flexible network architecture. The network middle layer, each level processing unit number as well as the network study parameter (study rate, study number of times, individual and global error) may be established willfully according to the special details. But the BP network has some problems that its convergence rate is slow. Regarding some complex questions, the BP algorithm possibly carry on the training of several hours even longer time. Especially the big sample recognition duty is a challenge to the BP network.

Design of neural network involves the selection of its model, architecture, learning algorithm, and activation function. For Palmprint recognition problem Back propagation neural network can be used with one or more hidden layer. Hidden layer is used to reduce complexity and increase computational efficiency.

\section{A. Input layer}

This layer takes the inputs (the values we pass) and forwards it to hidden layer. Input layer created for neural network is determined by characteristics of inputs. Here four attribute feature vector are obtained. So number of neurons in input layer is four.

\section{B. Hidden layer}

Hidden layer automatically extracts the feature of the input pattern [12]. There is no definite rule to find the number of neuron in hidden layer. So, it is hit and trial method. Where, network has to be tested with different neurons in hidden layer. Here it is found that 40 neurons in hidden layer could accomplish the task with good recognition rate.

For hidden layer hyperbolic tangent sigmoid transfer function is used. At the hidden layer it is used to calculate network output from its input. The tangent hyperbolic function and its fast approximation are given by following equation.

$$
a_{i 1}=\operatorname{tansig}\left(n_{i 1}\right)=\frac{2}{\exp \left(-2 n_{12}\right)+1}-1
$$

Where $\mathbf{a}_{\mathbf{i} 1}$ is the ith element of a1 vector is contains output from the hidden neurons. $\mathrm{n}_{\mathrm{i1}}$ is the ith element of $\mathrm{n} 1$ vector containing net input going into the hidden units. $\mathrm{n} 1$ is calculated by using the following formula.

$$
\mathrm{n}_{1}=\mathrm{w}_{10} \mathrm{p}+\mathrm{b}_{1}
$$

(6)

Where $\mathrm{p}$ is input pattern $\mathrm{b} 1$ is the vector of bias. $\mathrm{w}_{10}$ is the weight matrix between hidden layer and output layer. 


\section{Output Layer}

Output layer is designed based on the required output of neural network. Here 25 subject data are taken, so number of neurons in output layer is 25 . Pure linear activation function is selected for output, given by following equation.

$$
\mathrm{a}_{2}=\mathrm{n}_{2}
$$

Where $a_{2}$ is the column vector coming from output layer, $n_{2}$ is the output net inputs going into the output layer can be calculated by using following formula:

$$
\mathrm{n}_{2}=\mathrm{w}_{21} \mathrm{a}_{1}+\mathrm{b}_{2}
$$

Where b2 is the bias at second layer, w21 is the synaptic weights at hidden layer and output layer, a1 is the column vector.

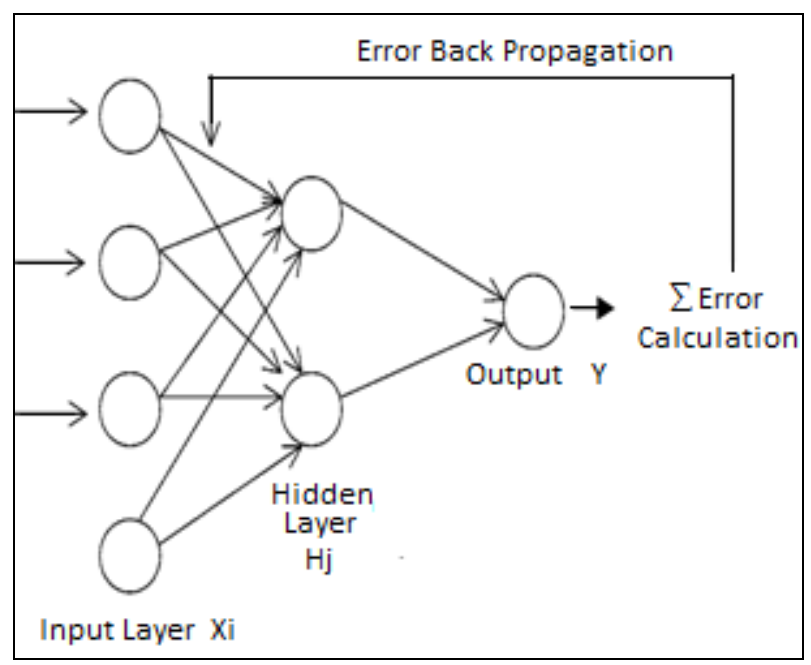

Fig. 4- neural network

\section{EXPERIMENTS AND RESULTS}

The paper uses the ANN architecture for feed forward back propagation learning algorithm to create, train and test the neural network for palm print recognition. In addition, MATLAB software with its neural network toolbox is used. In this analysis, the transfer function between input layer and hidden layer is tangent sigmoid function tansig, and one between hidden layer and output layer is linear function purelin.

The experiments are performed on the basis of database created.

Feed forward back propagation neural network are used for training and testing. Training algorithm is LevenbergMarquardt, performance is measure by mean square error, and data division is random. Results are marked as bold for the best performance. The results show that the best classification accuracy is $99.998 \%$ for two hidden layers. Fig. 6, Fig 7, Fig. 8 respectively show performance plot, regression plot and training state graph for highest performance.
Table.1 - Results

\begin{tabular}{|l|l|l|l|l|}
\hline $\begin{array}{l}\text { S. } \\
\text { no. }\end{array}$ & $\begin{array}{l}\text { Number } \\
\text { of hidden } \\
\text { layer }\end{array}$ & $\begin{array}{l}\text { No. of } \\
\text { Neuron }\end{array}$ & Performance & Epoches \\
\hline 1 & 1 & 10 & 98.62 & 14 \\
\hline 2 & 2 & 20 & 99.998 & 9 \\
\hline 3 & 3 & 50 & 95.81 & 6 \\
\hline
\end{tabular}

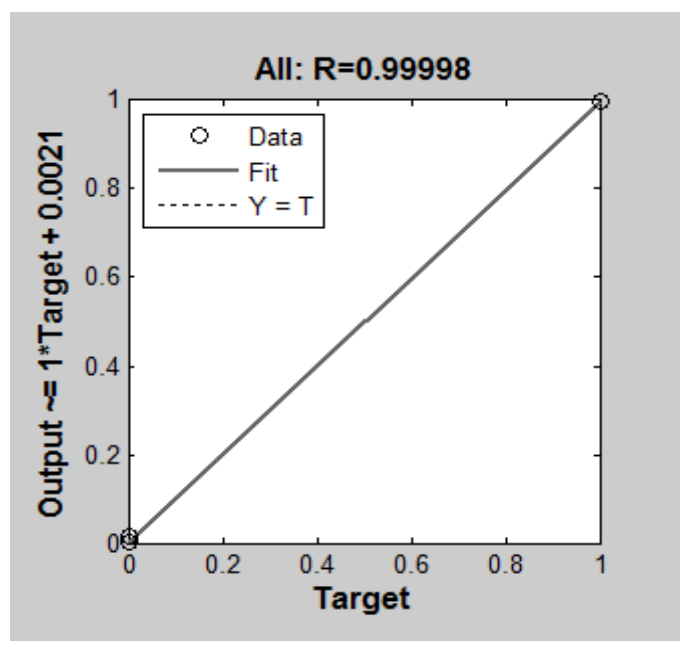

Fig.-6 - performance plot

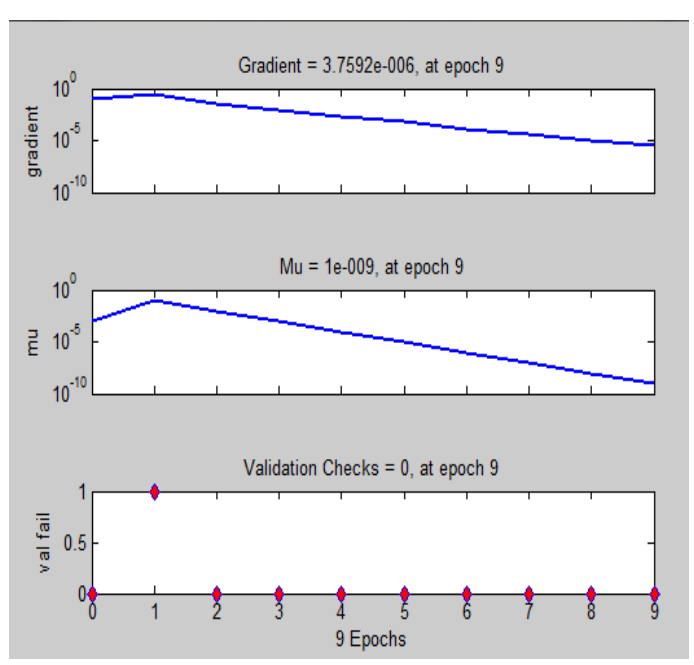

Fig.-7-regression plot

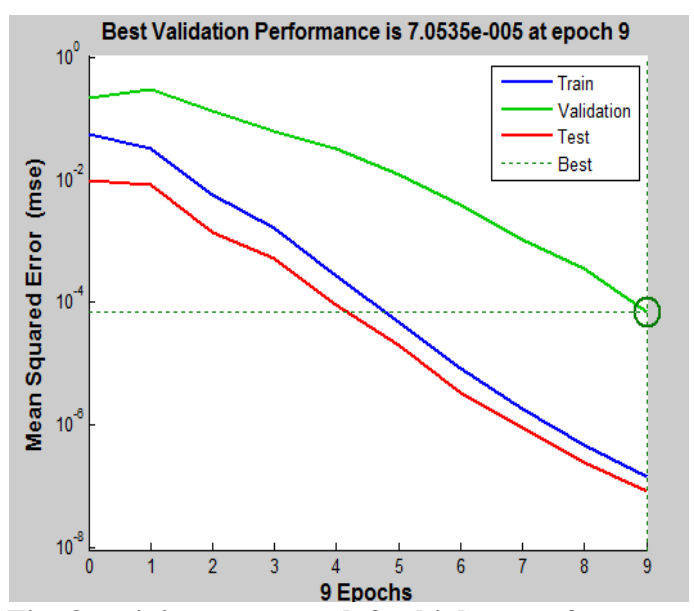

Fig.-8-training state graph for highest performance 


\section{CONCLUSION AND FUTURE WORK}

This paper proposes a new method for palm print based recognition. Image processing operations are applied to extract the features of palm. Neural network is used as classifier. The result shows that, features that are extracted here give the good accuracy results.

The work can be extended to develop a new biometric system in which, number of object can be divided into different section. So it can be use as one of the good reliable way of authentication.

\section{REFERENCES}

[1] Jiwen Lu,Yongwei Zhao,Yanxue Xue and Junlin $\mathrm{Hu}$ "Palm print Recognition via Locality Preserving Projections and Extreme Learning Machine Neural Network" ICSP2008 Proceedings.

[2] Xiaoxu Cheng and Shuhua Wang " A New Palmprint Identification Technique Based on a Two -stage Neural Network Classifier" 2008 International Conference on Computer Science and Software Engineering.

[3] You J., Li W., Zhang D. Hierarchical Palmprint Identification via Multiple Feature Extraction. Pattern Recognition,35, 847-859, 2002.

[4] Zhang, D., Shu, W.: Two Novel Characteristics in Palmprint verification: datum point invariance and line feature matching. Pattern Recognition Letters 32, 691702 (1999)

[5] Wu, X., Wang, K., Zhang, D.: Fuzzy directional element energy feature based palmprint identification. In: Proc. of International Conference on Pattern Recognition, vol. 1, pp. 95-98 (2002)

[6] Han, C.C., Cheng, H.L., Lin, C.L., Fan, K.C.: Personal Authentication Using Palmprint.Pattern Recognition 36, 281-371 (2003).
[7] Han C.C., Cheng H.L., Lin C.L., Fan K.C. Personal Authentication using Palmprint Features. Pattern Recognition,36:371-381,2003.

[8] Li, W., Zhang, D., Xu, Z.: Palmprint Identification by Fourier Transform. International Journal of Pattern Recognition and Artificial Intelligence 16, 417-432 (2003).

[9] Kong, W.K., Zhang, D., Li, W.: Palmprint Feature Extraction using 2-D Gabor Filters. Pattern Recognition 36, 2339-2347 (2003)

[10] Lu, G., Zhang, D., Wang, K.: Palmprint Recognition using Eigenpalms Features. Pattern Recognition Letters 24, 1473-1477 (2003)

[11] Wu, X., Zhang, D., Wang, K.: Fisherpalm Based Palmprint Recognition. Pattern Recognition Letters 24, 2829-2838 (2003)

[12] Shang, L., Zhang, D., Du, J., Zheng, C.: Palmprint Recognition Using FastICA algorithm and Radial Basis Probabilistic Neural Network. Pattern Recognition Letters 69, 1782- 1786 (2006).

[13] M. Wang, Q. Ruan, "Palmprint recognition based on two dimensional methods",Proceedings of ICSP, 2006.A. Kumar, D. Zhang, "Personal recognition using hand shape and texture", IEEE Transactions on Image Processing, vol. 15, no. 8,pp. 2454-2461, 2006.

[14] F. Li, M. K. H. Leung, X. Yu, "A two-level matching scheme for speedy and accurate palmprint identification”, LNCS 4352, pp.323-332, 2007.

[15] W-S Chen, Y-S Chiang, Y-H Chiu, "Biometric verification by fusing hand geometry and palmprint", Proceedings of Int. Conf. on Intelligent Information Hiding and Multimedia Signal Processing (IIHMSP), pp. 403-406, 2007.

[16] M. Ekinci, M. Aykut, "Palmprint recognition by applying wavelet subband representation and kernel PCA”, LNAI 4571, pp. 628-642, 2007.

[17] M. Ekinci, M. Aykut, "Palmprint recognition by applying wavelet based kernel PCA", J. of Computer Science, vol. 23, no. 5, pp.851-861, 2008 